\title{
Diagnostic Value of miR-106a-5p in Patients with Psoriasis and its Regulatory Role in Inflammatory Responses
}

Xiaolin Miao ( $\sim$ miaoxl2736@163.com )

Tongde Hospital Of Zhejiang Province https://orcid.org/0000-0002-6148-5027

\section{Xinyun Tong}

First Affiliated Hospital of Anhui Medical University

Jingsang Hu

Tongde Hospital Of Zhejiang Province

Juan Wang

Tongde Hospital Of Zhejiang Province

\section{Research}

Keywords: Psoriasis, miR-106a-5p, PASI, biomarker, proliferation

Posted Date: August 25th, 2020

DOl: https://doi.org/10.21203/rs.3.rs-60976/v1

License: (c) (i) This work is licensed under a Creative Commons Attribution 4.0 International License. Read Full License 


\section{Abstract}

Background: Psoriasis is a multifactorial, recurring, and chronic inflammatory skin disease. This study was designed to explore the potential role of microRNA-106a-5p (miR-106a-5p) in psoriasis.

Methods: The expression levels of miR-106a-5p in the serum of psoriasis patients and healthy individuals were detected by quantitative real-time polymerase chain reaction (qRT-PCR). The diagnostic value of miR-106a-5p in serum was evaluated by the receiver operating characteristics curve (ROC). The levels of interleukin-22 (IL-22), interleukin-17A (IL-17A), and tumor necrosis factor-alpha (TNF-alpha) were determined by enzyme-linked immunosorbent assay.

Results: The serum expression of miR-106a-5p was found to be up-regulated in psoriasis patients. ROC curve showed that miR-106a-5p had high specificity and sensitivity in the diagnosis of psoriasis. The correlation between the serum expression level of miR-106a-5p and PASI was positive. The relative expression levels of IL-17A, IL-22 and TNF-alpha in serum of psoriasis patients were significantly upregulated compared with that in healthy control, and showed positive association with serum miR106a-5p levels. Cell experiments demonstrated that upregulation of miR-106a-5p could promote cell proliferation, and the levels of IL-22, IL-17A and TNF-alpha were upregulated significantly in M5-induced HaCaT cells.

Conclusion: Considering the novel and vital role in psoriasis progression, miR-106a-5p is expected to be a new potent target for treatment of psoriasis. MiR-106-5p was expected to use for more immunity diseases research and therapy.

\section{Introduction}

Psoriasis, a chronic inflammatory disease of skin, is characterized by well-demarcated, raised, and scaling skin lesions [1, 2]. The interaction of epidermal cells and cytokines promotes the proliferation of abnormal keratinocytes, angiogenesis, and the development and maturation of psoriasis [3]. Psoriasis is a complex disorder requiring a genetic susceptibility and an environmental trigger [4]. It is commonly used as a model of immune-mediated diseases. The gene expression profile of the affected skin has made people deeply understand the pathogenesis of psoriasis [5]. However, the mechanism leading to psoriasis-specific mRNA expression is unclear.

MicroRNAs (MiRNAs), a class of small non-coding RNA molecules, are 18-25 nucleotides in length generally [6]. More and more evidence has confirmed the role of miRNAs in normal biological processes and human disease pathogenesis through post-transcriptional regulation of gene expression [7]. Some miRNAs have been found to have an effect on psoriasis [8-10]. Torri A et al. explored the differences in the expression levels of circulating microRNAs among patients with psoriasis and healthy individuals, in which the serum expression of miR-106a-5p was significantly increased [11]. Considering the aberrant expression levels of miR-106a-5p in psoriasis, the specific role of miR-106a-5p in psoriasis attracts our attention. However, the exact pathogenesis of psoriasis remains unclear. Therefore, it is a hot in psoriasis 
research to accurately predict the progress of disease and its therapeutic effect by finding characteristic biomarkers at present.

In our study, we hypothesized that miR-106a-5p could serve as a novel diagnostic marker in psoriasis.

We detected the expression profile of miR-106a-5p in psoriasis serum samples. MiR-106a-5p was identified to be upregulated in psoriasis patients, and had relative ability for the early diagnosis for psoriasis. Furthermore, the role of miR-106a-5p in inflammatory responses in keratinocytes were investigated.

\section{Materials And Methods}

\section{Patients}

67 patients with psoriasis who had no previous treatment for psoriasis (mean age: $43.33 \pm 12.16$ years) and 70 healthy subjects (mean age: $48.37 \pm 14.99$ years) were recruited from September 2015 to January 2018 in Tongde Hospital of Zhejiang Province. Patients with cardiovascular and cerebrovascular diseases, autoimmune diseases and other serious systemic diseases, pregnancy, lactation and so on were excluded. To evaluate disease type and severity, patients were all examined by dermatologist. Psoriasis Area Severity Index (PASI) scores were used. Individuals with PASI index ranging from 1 to 10 were considered as mild disease group, patients with PASI > 10 as moderate or severe group [12].

Informed consent was obtained from every patient with psoriasis and healthy individuals, and this study was approved by the Ethics Committee of Tongde Hospital of Zhejiang Province.

\section{Cell culture}

Human keratinocytes HaCaT cells were purchased from Cell Lines Service (Eppelheim, Germany). The cells were cultured in Dulbecco's modified Eagle's medium (DMEM; Invitrogen, Carlsbad, CA, USA) supplemented with 10\% fetal bovine serum (FBS; Sigma-Aldrich, St Louis, MO, USA), and placed in a humidified atmosphere of $5 \% \mathrm{CO}_{2}$ at $37^{\circ} \mathrm{C}$. TNF-alpha, IL-17A, IL-22, IL-1 alpha, and Oncostatin-M $(10 \mathrm{ng} / \mathrm{mL}$, Prospec, East Brunswick, NJ) were included in the mixture of five proinflammatory cytokines (M5) [13]. HaCaT cells were induced with M5, causing inflammation response and showing kinds of symptoms of psoriasis. The culture medium was changed every day. The cells were disaggregated and sub-cultured at a confluency of $70-90 \%$.

\section{Cell transfection}

HaCaT cells were transfected with miR-106a-5p mimic, miR-106a-5p inhibitor and its negative control (miR-NC) using the Lipofectamine 2000 reagent (Invitrogen) in accordance with manufacturer protocols. The miR-106a-5p mimic, inhibitor and Negative Control were obtained from GenePharma (Shanghai, China). The qRT-PCR was performed for measuring the transfection efficiency. After $24 \mathrm{~h}$ transfection, 
HaCaT cells were treated with M5 (a cocktail of cytokines) for $24 \mathrm{~h}$ to induce psoriatic inflammation like condition.

Cell Culture

\section{qRT-PCR assay}

According to the manufacturer's instructions, total RNA was isolated using TRIzol reagent (Invitrogen, USA). MiR-106a-5p was detected by reverse transcription of miRNA using a TaqMan MicroRNA Reverse Transcription Kit (Applied Biosystems, CA, USA), and measured by RT-PCR with the TaqMan MicroRNA Assay kit (Applied Biosystems, CA, USA) [14]. U6 small nuclear RNA (U6 snRNA) was quantified as an endogenous reference. Experiments were performed in triplicate and run at least three times.

\section{Enzyme-linked immunosorbent assay (ELISA)}

Reference to the instructions of the manufacturer, the serum levels of IL-22, IL-17A, and TNF-alpha were detected by specific enzyme-linked immunosorbent (ELISA) kits (Beyotime Biotechnology, Shanghai, China). The absorbance was determined by enzyme labeling instrument (spectraMAX 340, Molecular Devices, Sunnyvale, CA, USA) at $450 \mathrm{~nm}$.

\section{Cell proliferation assay}

The CCK-8 assay was performed to determine the effect of miR-106a-5p on the proliferation of HaCaT cells. Briefly, cells were seeded in 96 -well plates at the density of $1 \times 10^{4}$ per well. After 24,48 and $72 \mathrm{~h}, 20$ $\mu \mathrm{L}$ of CCK-8 reagent were added into each well, and then incubated for $2 \mathrm{~h}$ at $37^{\circ} \mathrm{C}$. After all the treatment, the optical density values (OD) of each well was measured by a Microplate Reader (Bio-Rad, USA) at a wavelength of $450 \mathrm{~nm}$, which could exhibit the proliferation capacity of the cells. The experiment was performed in triplicate.

\section{Statistical analysis}

The experimental data was expressed as mean \pm standard deviation (SD). Statistical analysis of all data was used the SPSS 24.0 (SPSS Inc., Chicago, IL, USA) and GraphPad Prism 7.04 software (GraphPad, San Diego, CA, USA). The statistical differences between groups were compared by student's t-test and one-way ANOVA analysis. Pearson's correlation coefficient was applied for the correlation analysis. $P<$ 0.05 was considered as a statistically significant criterion. Each experiment run at least three times.

\section{Results}

\section{Increased serum levels of miR-106a-5p in psoriasis patients}

The expression level of miR-106a-5p in the serum of healthy subjects and psoriasis patients was detected by the method of qRT-PCR. The result demonstrated that the expression level of miR-106a-5p in the serum of psoriasis patients significantly increased compared with the healthy controls (Fig. 1, P<0.001). 


\section{miR-106a-5p was correlated with the psoriasis area and severity index (PASI) in psoriasis patients}

The correlation analyses between the serum expression level of miR-106a-5p and psoriasis activity was performed. Psoriasis activity was evaluated by the psoriasis area and PASI score, and we found a significantly positive association between PASI scores with the serum expression level of miR-106a-5p (Fig. 1B; Pearson's $r=0.6187 ; P<0.001$ ).

\section{Diagnostic potential of serum miR-106a-5p levels in psoriasis patients}

To assess the diagnostic potential of serum miR-106a-5p levels in psoriasis patients, the receiver operating characteristic (ROC) curve was established. As Fig. 2 showed that miR-106a-5p could discriminate the psoriasis patients from the healthy patients. The area under the curve (AUC) for miR$106 a-5 p$ was 0.901 with a sensitivity of $74.6 \%$ and specificity of $91.7 \%$ at the cutoff value of 1.195 .

\section{Association of miR-106a-5p with inflammatory factors}

Firstly, we detected the protein expression levels of IL-22, IL-17A and TNF-alpha. As shown in Fig. 3, the protein levels of IL-17A (Fig. 3A), IL-22(Fig. 3B) and TNF-alpha (Fig. 3C) in psoriasis group was significantly increased compared with that in control group $(P<0.001)$.

Additionally, the correlations between the serum expression level of miR-106a-5p and inflammatory factors (IL-22, IL-17A and TNF-alpha) were analyzed. We found that serum levels of miR-106a-5p were positively correlated with the protein levels of IL-17A (Fig. 3D; Pearson's $r=0.7017 ; P<0.001$ ), IL-22 (Fig. 3E; Pearson's $r=0.6740 ; P<0.001$ ) and TNF-alpha (Fig. 3F; Pearson's $r=0.7721 ; P<0.001$ ).

\section{miR-106a-5p regulated HaCaT cells proliferation and inflammatory response in vitro}

To explore the effects of miR-106a-5p in psoriasis, we transfected HaCaT cells with a miR-106a-5p mimic to upregulate miR-106a-5p ( $P<0.05$; Fig. 4A). The CCK-8 assay was used to detect the effect of miR$106 a-5 p$ on the proliferation of M5-induced HaCaT cells. Further analysis demonstrated that miR-106a-5p mimic can significantly increase the proliferation of M5-stimulated HaCaT cells, while miR-106a-5p inhibitor can significantly inhibit the proliferation of cells $(P<0.001$, Fig. 4B). These data suggested that miR-106a-5p regulated M5-induced keratinocyte proliferation.

To further study the effect of miR-106a-5p on the expression of inflammation factor in M5-induced HaCaT cells, we detected the protein expression levels of IL-22, IL-17A and TNF-alpha in cells. After transfecting by miR-106a-5p mimic, the levels of IL-22, IL-17A and TNF-alpha were upregulated significantly in M5-induced HaCaT cells (Fig. 4C, P < 0.001). And miR-106a-5p downregulation had the opposite effect (Fig. 4C, $\mathrm{P}<0.001$ ). 


\section{Discussion}

Psoriasis, an immune-mediated chronic inflammatory skin disease, is featured with epidermal hyperplasia, angiogenesis, and inflammatory cell infiltration $[15,16]$. It is a key psoriasis treatment to inhibit these cellars events. Evidences indicated that numerous internal and external factors might participate in psoriasis pathogenesis, including the immune system, environmental factors, keratinocytes and susceptibility genes [17]. Owing to the complexity of the cause of psoriasis, its pathogenesis has not yet been fully understood.

Increasing evidence has shown that abnormally expressed microRNA (miRNA) takes effects on psoriasis progression, such as miR-146a [18], miR-125b [19] and miR-135b [20]. These dysregulated miRNAs play an important role in diagnosis and treatment and have attracted increasing attention for their therapeutic potential in diseases. For instance, in psoriasis patients, the level of miR-205-5p was downregulated, and played its roles by targeting Ang-2, VEGFA and BAMBI, and deactivating the Wnt/ $\beta$-catenin and MAPK signaling pathways, which provided a potential therapeutic target for clinical treatment of psoriasis [21]. Work by Wang et al. established that miR-223 was overexpressed in psoriatic lesions and in IL-22stimulated $\mathrm{HaCaT}$ cells, and increased proliferation and inhibited apoptosis of IL-22-stimulated keratinocytes via the PTEN/Akt pathway [8]. Another study demonstrated that miR-876-5p was downregulated in psoriasic tissue and blood of patients and would be expected to be biomarker and potential therapeutic targets for the treatment of psoriasis [22]. In this study, the serum expression level of miR$106 a-5 p$ in psoriasis patients was higher than that in healthy control, which was consistent with two previous studies $[5,11]$. We further studied the correlation between miR-106a-5p and PASI score. Experimental results demonstrated the correlation between the expression levels of miR-485-5p and PASI score were positive significantly, which indicated a probable connection between psoriasis progression and miR-106a-5p. These results motivated us to study the role of miR-106a-5p in psoriasis further.

MiR-106a-5p was reported to be dysregulated in various of diseases, such as prostate cancer[23], atherosclerosis [24] and cervical cancer [25]. The clinical value of miR-106a-5p in diseases attracted researcher attention. As reported by Zhang et al., miR-106a-5p level was downregulated in osteoarthritis (OA) samples and IL-1b-treated chondrocytes, miR-106a-5p overexpression led to proliferation promotion and apoptosis inhibition in chondrocytes treated by IL-1 $\beta[26]$. Another study in HPV-16-associated cervical cancer reported that miR-106a-5p played an important role in regulatory mechanism of cervical squamous cell carcinoma (CSCC) and could be a potential therapeutic target in HPV-associated cervical cancer [25]. Considering the dysregulation of miR-106a-5p in the serum of psoriasis patients, we further explored whether serum miR-106a-5p could differentiate between the psoriasis patients and the healthy. The area under the ROC curve (AUC) of miR-106a-5p on diagnosis of psoriasis was 0.901 with a sensitivity of $74.60 \%$ and specificity of $91.7 \%$ at the cutoff value of 1.195 , indicating miR-106a-5p has ability to distinguish the psoriasis patients from healthy control.

In this study, it was found that the protein levels of IL-17A, IL-22 and TNF- alpha were significantly increased in serum. The result demonstrated that inflammation play an important role in psoriasis. We 
further explored the correlations between the serum expression level of miR-106a-5p and inflammation cytokines. Results indicated that serum levels of miR-106a-5p were positively correlated with the protein levels of IL-22, IL-17A and TNF-alpha. Therefore, it came to this conclusion that upregulated miR-106a-5p might promote the occurrence and development of psoriasis through promoting inflammatory response.

HaCaT cells are immortalized cells, which are commonly used in the studies relevant to psoriasis [13]. To mimic conditions of psoriatic keratinocytes, HaCaT cells were induced with M5 (consisting of IL-17A, IL22, IL-1 alpha, TNF-alpha and Oncostatin M) as previous reported [27]. Therefore, we established a cell model of highly proliferative psoriatic keratinocytes via treating HaCaT cells by $\mathrm{M} 5$. It is found that the expression level of miR-106a-5p was apparently increased in M5-treated HaCaT cells, which was consistent with the results observed in the serum of psoriasis patients. Meanwhile, the upregulation of miR-106a-5p in M5-treated HaCaT cells significantly promoted its proliferation. Additionally, the protein levels of inflammation cytokines (IL-17A, IL-22 and TNF- alpha) were all increased significantly in M5treated $\mathrm{HaCaT}$ cells. These results suggested that miR-106a-5p might be a potential treatment target in psoriasis.

In conclusion, our work demonstrates that miR-106a-5p had a high expression in psoriasis patients and can distinguish psoriasis patients from healthy controls. MiR-106a-5p might be involved in the disease progression through regulating inflammatory response. Our results provide new insight of miR-106a-5p and may contribute to the development of target for psoriasis treatment.

\section{Declarations}

\section{Ethics approval and consent to participate}

Informed consent was obtained from every patient with psoriasis and healthy individuals, and this study was approved by the Ethics Committee of Tongde Hospital of Zhejiang Province.

\section{Consent for publication}

Written informed consent for publication was obtained from each participant.

\section{Availability of data and materials}

The datasets used and/or analysed during the current study are available from the corresponding author on reasonable request.

\section{Competing interests}

The authors declare that they have no competing interests. 


\section{Funding}

Not applicable.

\section{Authors' contributions}

Xiaolin Miao, Xinyun Tong, Jingsang Hu, and Juan Wang initiated and designed the work, analyzed data, wrote and revised the manuscript. Xiaolin Miao and Xinyun Tong contributed to acquisition of patients and tissues specimens, interpretation of data. All authors read and approved the final manuscript.

\section{Acknowledgements}

Not applicable.

\section{References}

1. Gupta R, Debbaneh MG, Liao W. Genetic Epidemiology of Psoriasis. Curr Dermatol Rep. 2014;3(1):61-78.

2. Vicentini FT, Borgheti-Cardoso LN, Depieri LV, de Macedo Mano D, Abelha TF, Petrilli R, et al. Delivery systems and local administration routes for therapeutic siRNA. Pharm Res. 2013;30(4):915-31.

3. Zhang F, Li H, Zhou Y, Gu Y, Wang L. Caveolin-1 expression in different types of psoriatic lesions: analysis of 66 cases. Indian J Dermatol. 2014;59(3):225-9.

4. Elder JT, Nair RP, Henseler T, Jenisch S, Stuart P, Chia N, et al. The genetics of psoriasis 2001: the odyssey continues. Arch Dermatol. 2001;137(11):1447-54.

5. Delic D, Wolk K, Schmid R, Gabrielyan O, Christou D, Rieber K, et al. Integrated microRNA/mRNA expression profiling of the skin of psoriasis patients. J Dermatol Sci. 2020;97(1):9-20.

6. Cai MY, Cheng J, Zhou MY, Liang LL, Lian SM, Xie XS, et al. The association between pre-miR-27a rs895819 polymorphism and myocardial infarction risk in a Chinese Han population. Lipids Health Dis. 2018;17(1):7.

7. Rupaimoole R, Slack FJ. MicroRNA therapeutics: towards a new era for the management of cancer and other diseases. Nat Rev Drug Discov. 2017;16(3):203-22.

8. Wang R, Wang FF, Cao HW, Yang JY. MiR-223 regulates proliferation and apoptosis of IL-22stimulated HaCat human keratinocyte cell lines via the PTEN/Akt pathway. Life Sci. 2019;230:2834.

9. Duan Y, Zou J, Mao J, Guo D, Wu M, Xu N, et al. Plasma miR-126 expression correlates with risk and severity of psoriasis and its high level at baseline predicts worse response to Tripterygium wilfordii Hook F in combination with acitretin. Biomed Pharmacother. 2019;115:108761. 
10. Zhang D, Wang Y, Xia Y, Huo J, Zhang Y, Yang P, et al. Repression of miR-142-3p alleviates psoriasislike inflammation by repressing proliferation and promoting apoptosis of keratinocytes via targeting Sema3A. Mol Cell Probes. 2020;52:101573.

11. Torri A, Carpi D, Bulgheroni E, Crosti MC, Moro M, Gruarin P, et al. Extracellular MicroRNA Signature of Human Helper T Cell Subsets in Health and Autoimmunity. J Biol Chem. 2017;292(7):2903-15.

12. Debniak T, Soczawa E, Boer M, Rozewicka-Czabanska M, Wisniewska J, Serrano-Fernandez P, et al. Common variants of ZNF750, RPTOR and TRAF3IP2 genes and psoriasis risk. Arch Dermatol Res. 2014;306(3):231-8.

13. Kim HR, Kang SY, Kim HO, Park CW. B. Y. Chung. Role of Aryl Hydrocarbon Receptor Activation and Autophagy in Psoriasis-Related Inflammation. Int J Mol Sci. 2020;21(6).

14. Sun X, Liu S, Wang J, Wei B, Guo C, Chen C, et al. Annexin A5 regulates hepatocarcinoma malignancy via CRKI/II-DOCK180-RAC1 integrin and MEK-ERK pathways. Cell Death Dis. 2018;9(6):637.

15. Xiao $Y$, Wang $H$, Wang $C$, Zeng $B$, Tang $X$, Zhang $Y$, et al. miR-203 promotes HaCaT cell overproliferation through targeting LXR-alpha and PPAR-gamma. Cell Cycle. 2020:1-13.

16. Teng $X$, Hu Z, Wei $X$, Wang Z, Guan T, Liu N, et al. IL-37 ameliorates the inflammatory process in psoriasis by suppressing proinflammatory cytokine production. J Immunol. 2014;192(4):1815-23.

17. Boehncke WH. M. P. Schon Psoriasis Lancet. 2015;386(9997):983-94.

18. Kocic H, Damiani G, Stamenkovic B, Tirant M, Jovic A, Tiodorovic D, et al. Dietary compounds as potential modulators of microRNA expression in psoriasis. Ther Adv Chronic Dis. 2019;10:2040622319864805.

19. Xu N, Brodin P, Wei T, Meisgen F, Eidsmo L, Nagy N, et al. MiR-125b, a microRNA downregulated in psoriasis, modulates keratinocyte proliferation by targeting FGFR2. J Invest Dermatol. 2011;131(7):1521-9.

20. Chicharro P, Rodriguez-Jimenez P, Llamas-Velasco M, Montes N, Sanz-Garcia A, Cibrian D, et al. Expression of miR-135b in Psoriatic Skin and Its Association with Disease Improvement. Cells. 2020;9(7).

21. Xue Y, Liu Y, Bian X, Zhang Y, Li Y, Zhang Q, et al. miR-205-5p inhibits psoriasis-associated proliferation and angiogenesis: Wnt/beta-catenin and mitogen-activated protein kinase signaling pathway are involved. J Dermatol. 2020.

22. Yu RA,P, Hao S, Li Y. MiR-876-5p suppresses cell proliferation by targeting Angiopoietin-1 in the psoriasis. Biomed Pharmacother. 2018;103:1163-9.

23. Wei P, Yang J, Zhang D, Cui M, Li L. IncRNA HAND2-AS1 Regulates Prostate Cancer Cell Growth Through Targeting the miR-106a-5p/RBM24 Axis. Onco Targets Ther. 2020;13:4523-31.

24. Hu Y, Xu R, He Y, Zhao Z, Mao X, Lin L, et al. Downregulation of microRNA106a5p alleviates oxLDLmediated endothelial cell injury by targeting STAT3. Mol Med Rep. 2020;22(2):783-91.

25. Cui X, Wang X, Zhou X, Jia J, Chen H, Zhao W. miR-106a regulates cell proliferation and autophagy by targeting LKB1 in HPV-16-associated cervical cancer. Mol Cancer Res. 2020. 
26. Zhang X, Liu X, Ni X, Feng P, Wang YU. Long non-coding RNA H19 modulates proliferation and apoptosis in osteoarthritis via regulating miR-106a-5p. J Biosci. 2019;44(6).

27. Kim HK, Bae MJ, Lim S, Lee W. S. Kim. A Water-Soluble Extract from Actinidia arguta Ameliorates Psoriasis-Like Skin Inflammation in Mice by Inhibition of Neutrophil Infiltration. Nutrients. 2018;10(10).

Figures
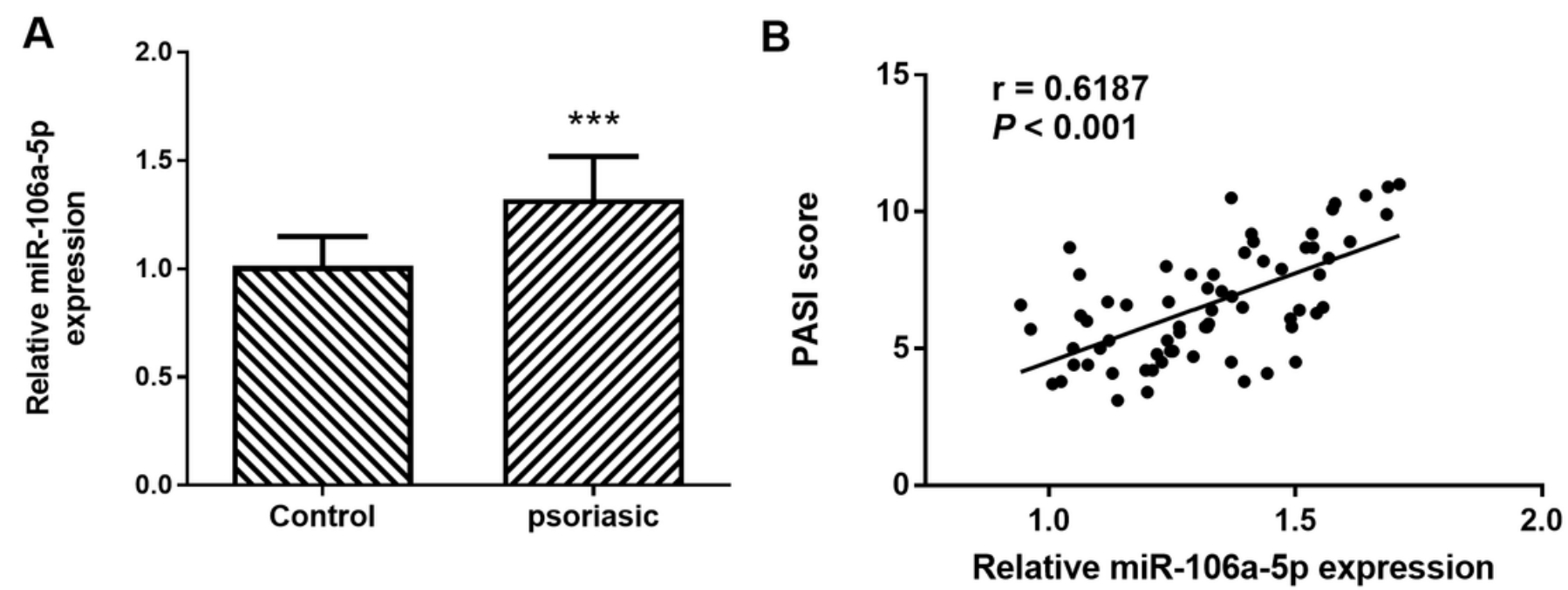

Figure 1

The expression levels of serum miR-106a-5p is related to disease severity of psoriasis. A. Serum miR106a-5p levels were significantly higher in psoriasis patients than that in healthy controls. $* \star * P<0.001$, compared with healthy controls. B. The correlation between the serum expression level of miR-106a-5p and PASI was positive. PASI, psoriasis area and severity index. 


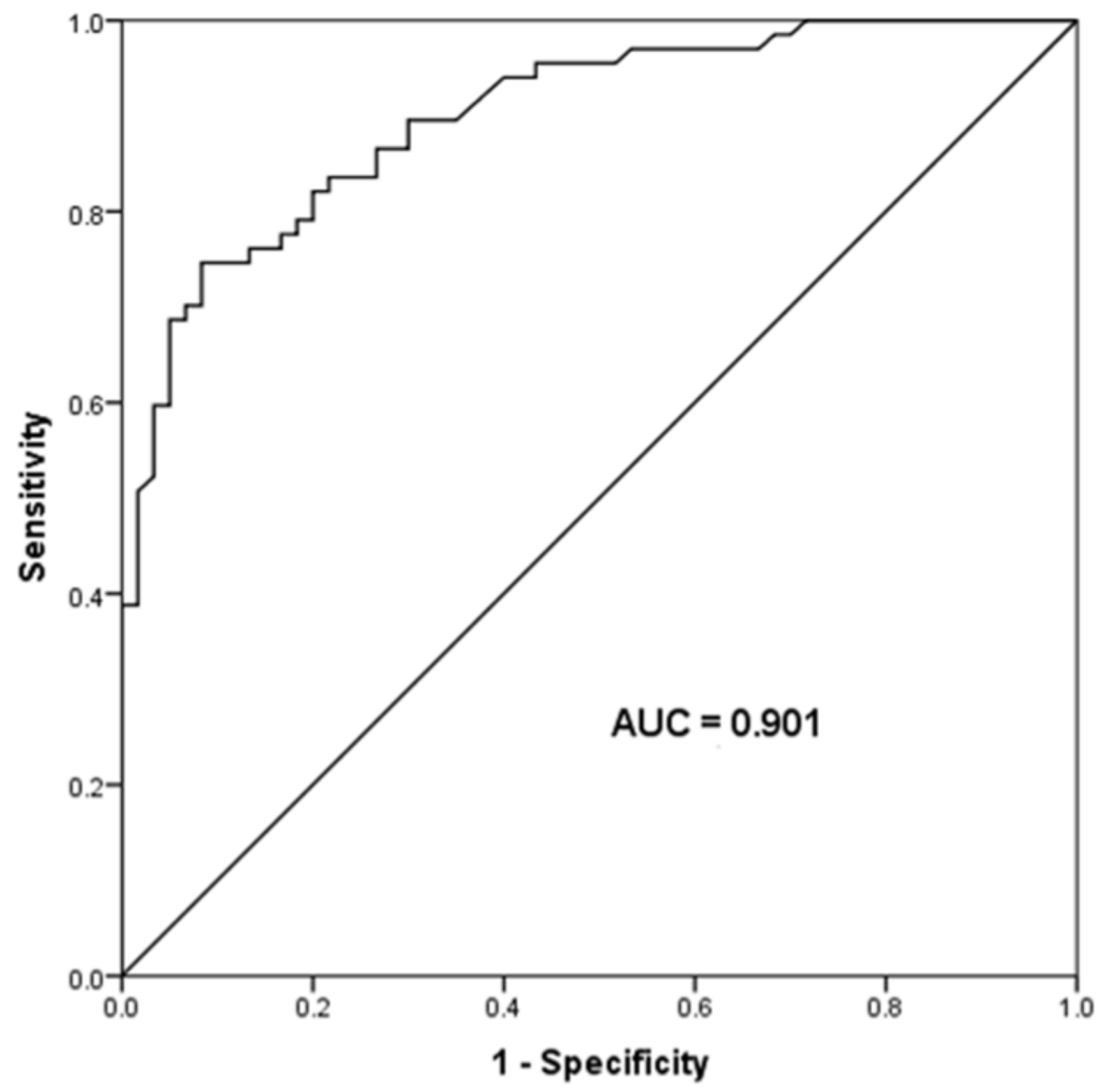

Figure 2

ROC curve was performed to assess the diagnostic value of serum miR-106a-5p in psoriasis patients and healthy control. 
A

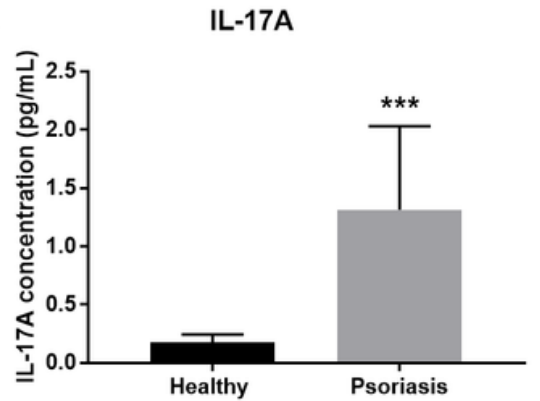

D

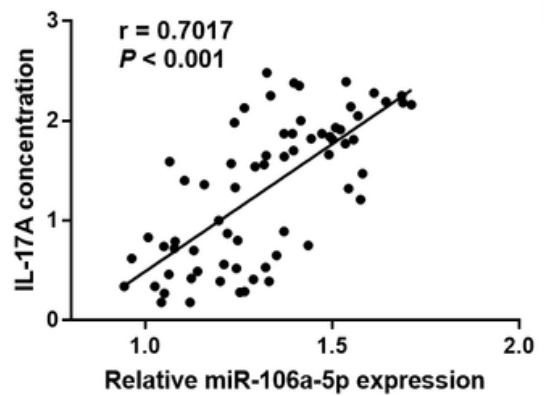

B

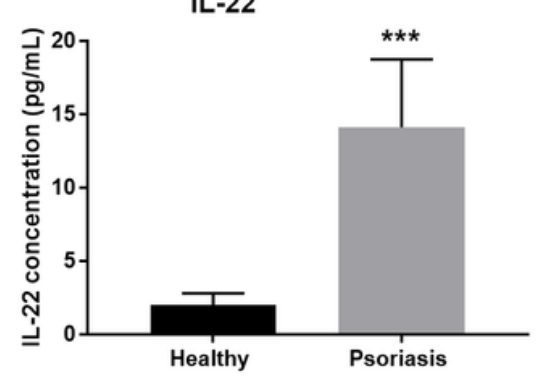

E

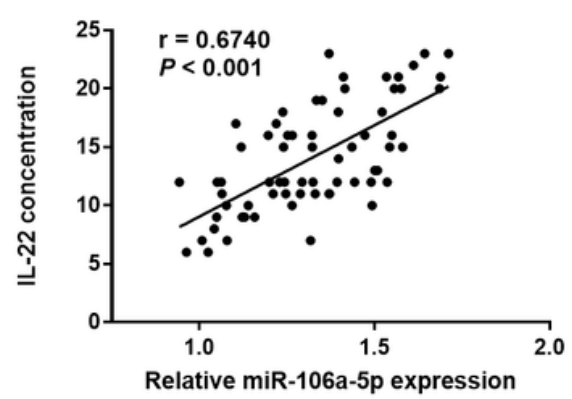

C

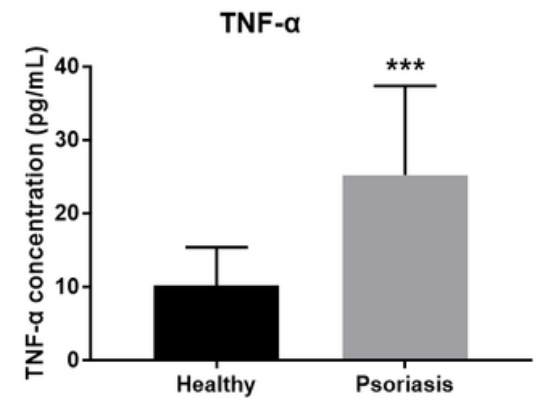

$\mathbf{F}$

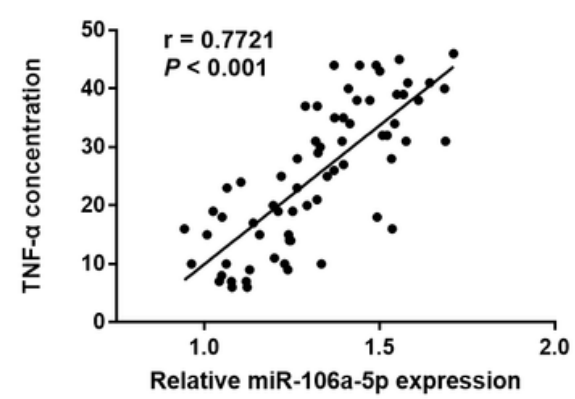

Figure 3

The relation between the serum expression level of miR-106a-5p and inflammation factor (IL-17A, IL-22 and TNF-alpha) were analyzed. A-C. The relative expression levels of IL-17A (), IL-22 and TNF-alpha in psoriasis patients were all significantly upregulated compared with that in healthy control $(P<0.001)$. *** $P<0.001$, compared with control group. D-F. Correlation between the expression level of miR-106a-5p and serum IL-17A, IL-22 and TNF-alpha levels.
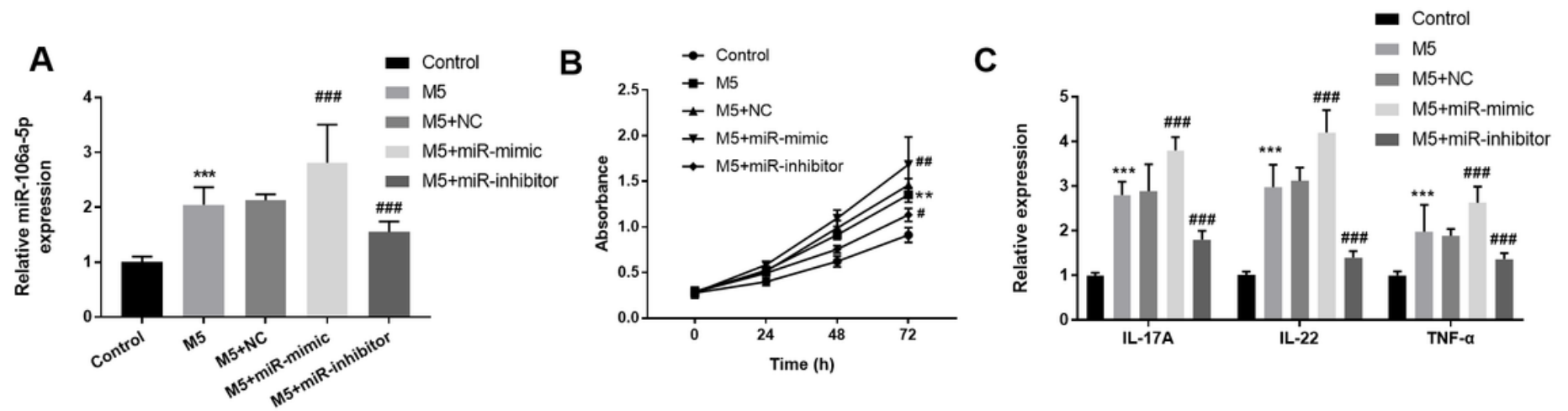

Figure 4

Effects of miR-106a-5p on proliferation of HaCaT cells. A. Changes in miR-106a-5p levels in cells after transfection with miR-106a-5p mimic and inhibitors were detected by qRT-PCR. ** $P<0.01$, compared with control group. \#\# P<0.01, \# P $<0.05$, compared with M5 group. B. Cell proliferation was detected by CCK-8 assay. miR-106a-5p mimic significantly promoted cell proliferation, while miR-106a-5p inhibitors 
significantly inhibited cell proliferation. C. The inflammatory cytokines levels in HaCaT cells were determined by enzyme-linked immunosorbent assay. *** $\mathrm{P}<0.001$, compared with control group. \#\#\# $\mathrm{P}<$ 0.001, compared with M5 group. 